\title{
Epidemiologic Evidence for the Development of Serovar-specific Immunity after Gonococcal Infection
}

\author{
F. A. Plummer, J. N. Simonsen, H. Chubb, L. Slaney, J. Kimata, M. Bosire, J. O. Ndinya-Achola, and E. N. Ngugi \\ Departments of Medical Microbiology and Medicine, University of Manitoba, Winnipeg, Manitoba, Canada R3E OW3; Center for \\ Microbiology Research, Kenya Medical Research Institute, Nairobi, Kenya; and Department of Medical Microbiology, \\ University of Nairobi, Nairobi, Kenya
}

\begin{abstract}
We tested the hypothesis that strain-specific immunity occurs after gonococcal infection in a longitudinal study of 227 prostitutes resident in one small community who experienced frequent gonococcal infections. Women were examined and cultured for Neisseria gonorrhoeae at 2-wk intervals. Gonococcal isolates were typed according to protein 1 serovar, auxotype, and $\beta$-lactamase plasmid type, and classified as to serovar and strain. The hypothesis was tested by comparing the predictions of the hypothesis with the observations of the study. Over the 14-mo period of the study, major changes in the prevalence of specific serovars were observed in the gonococcal population infecting these women. Women with HIV infection experienced a higher rate of gonococcal infection $(0.56 \pm 0.03$ vs. $0.46 \pm 0.04, P<0.05, t$ test) compared with HIV-negative women and were more likely to experience multiple infections with the same strain. The duration of prostitution was inversely related to the frequency of gonococcal infection. Women experiencing an infection with a specific gonococcal serovar were at a 2- to 10-fold reduced risk of reinfection with the same serovar, except for the 1B-1 serovar. The results of the study were consistent with all four predictions of the hypothesis. Infection with a specific gonococcal serovar results in specific but incomplete protection against subsequent infection with the homologous serovar. The mechanism of this protection remains to be determined.
\end{abstract}

\section{Introduction}

Although much studied, the role of immunity in gonococcal infection is uncertain. One of the certainties concerning any putative immunity to Neisseria gonorrhoeae is that after a gonococcal infection, the infected person may experience many subsequent infections. Although antibody to various surface proteins on $N$. gonorrhoeae can be demonstrated in individuals experiencing infection $(1,2)$, and can be shown to be bactericidal (3) (in the case of antibody to protein 1) or to block attachment to human cells $(4,5)$ (in the case of antibody

This work was presented before the International Society for Sexually Transmitted Disease Research, Atlanta, GA, August 1987.

Address correspondence to Dr. F. A. Plummer, Department of Medical Microbiology, University of Manitoba, 730 William Avenue, Winnipeg, Manitoba, Canada R3E 0W3.

Received for publication 4 February 1988 and in revised form 7 December 1988.

\footnotetext{
J. Clin. Invest.

(c) The American Society for Clinical Investigation, Inc.

$0021-9738 / 89 / 05 / 1472 / 05 \$ 2.00$

Volume 83, May 1989, 1472-1476
}

to pilus), there is no clear role for such antibodies in protection against gonococcal infection.

In considering how gonococci interact with human populations (the ecology of $N$. gonorrhoeae), two conclusions seem inescapable. First, humans must have evolved natural defence mechanisms against a pathogen that can so profoundly affect reproduction. Second, that $N$. gonorrhoeae must have evolved mechanisms for evading these defences, to continue to coexist with its obligate host. Over the past decade, our understanding of part of the ecologic relationships between $N$. gonorrhoeae and humans has advanced considerably. We now know that there is great diversity among gonococci and that this diversity is dynamic (6-8). We have also learned that within human populations certain segments $(9,10)$ (termed high frequency transmitters) are responsible for continued endemicity of $N$. gonorrhoeae in human communities.

We have advanced the hypothesis that the diversity and dynamism of gonococcal populations are ecologic defence mechanisms that allow $N$. gonorrhoeae to continue to exist in human populations that are continually developing immunity to $N$. gonorrhoeae (11). According to the hypothesis, protective strain-specific immunity develops after a natural gonococcal infection, ultimately resulting in immunity of the high frequency transmitter population to the specific gonococcal strains. In the face of this population immunity, a given gonococcal strain dies out. New strains must be continually evolving for $N$. gonorrhoeae to persist in the population. This hypothesis has been expressed by others as well (12). In this report we demonstrate that infection with a specific gonococcal serovar results in protection against subsequent infection with that serovar in a human high frequency transmitter population.

\section{Methods}

In January-March 1985, we established a cohort of prostitutes in a lower socioeconomic region of Nairobi, Kenya. These women had a known high prevalence and incidence of sexually transmitted disease (13). Details of the recruitment process and the characteristics of the study population have been published elsewhere (Simonsen, J. N., et al. manuscript submitted for publication). A subgroup of 227 women from the entire cohort of women were enrolled in a longitudinal study of gonococcal infection, beginning in March 1985. Women were followed at 2-wk intervals during the periods of the year they were resident in Nairobi. At each visit, a history, general physical exam, and complete genital examination were performed. Endocervical swabs for culture of $N$. gonorrhoeae were obtained. A follow-up appointment for treatment of infection was made 3 or $4 \mathrm{~d}$ later, at which time treatment was administered. $4 \mathrm{~d}$ after treatment a test of cure culture was performed.

Treatment for $N$. gonorrhoeae varied throughout the study period. Ceftriaxone, $125 \mathrm{mg}$ i.m., and spectinomycin, $2 \mathrm{~g}$ i.m., were the most frequently used treatments. Kanamycin, 1 g i.m., trimethoprim-sulfa- 
metrole, $400-2,000 \mathrm{mg}$ for $2 \mathrm{~d}$, and aqueous procaine penicillin $\mathrm{G}, 4.8$ million $\mathrm{U}$ with $1 \mathrm{~g}$ probenecid and $125 \mathrm{mg}$ clavulanic acid were also used. All regimens are effective for $N$. gonorrhoeae.

Laboratory methods. Swabs for $N$. gonorrhoeae were directly plated on modified Thayer-Martin media and transported to the laboratory within $3 \mathrm{~h}$ of inoculation. Cultures were incubated in humidified candle extinction jars at $37^{\circ} \mathrm{C}$ for $48 \mathrm{~h}$ before reading. $N$. gonorrhoeae was identified by colony morphology, oxidase reactivity, and gram stain morphology. Strains were subcultured once after picking multiple colonies, stocked in skim milk with $10 \%$ glycerol, and frozen at $-70^{\circ} \mathrm{C}$.

Gonococcal isolates were phenotypically characterized by auxotype, using standard methodology (14). Protein 1 serovar was determined using a panel of 12 MAbs to protein 1 as described by Knapp and Sandstrom (15). $\beta$-Lactamase production, plasmid content of strains, and minimal inhibitory concentrations of antimicrobial agents were performed as previously described (8). Antibody to HIV was determined by Dupont HTLV-III, ELISA, and Western blots (12).

Definitions. A gonococcal serovar is its protein 1 type as determined by the Knapp-Sandstrom MAb system. We defined a gonococcal strain as a unique combination of serovar, auxotype, and $\beta$-lactamase plasmid type. An episode of gonococcal infection was defined as the isolation of $N$. gonorrhoeae from the endocervix. A reinfection was considered to have occurred when an additional gonococcal infection was documented after an intervening negative test of cure culture or, in the event that a test of cure culture was not performed, the reinfection occurred within $2 \mathrm{wk}$ of the prior episode and was of a different gonococcal strain type, or if test of cure culture was positive but of a different gonococcal strain type. Subsequent infections were considered treatment failures if tests of cure cultures were positive and of the same strain type or, in the event that test of cure was not performed, if infection with the same strain was detected within $2 \mathrm{wk}$ of the preceding infection. There were 20 instances in which test-of-cure cultures were not performed; 16 were classified as treatment failures (serovars of isolates classified as treatment failures were $1 \mathrm{~B}-1,4$; 1 B-5, 1; A1-3, 5; $1 \mathrm{~A}-4,2$; and $1 \mathrm{~A}-6,4)$ and four were classified as reinfections on the basis of the above criteria.

Analysis of data. The number of episodes of infection in each individual was determined. Serovar-specific reinfection rates were determined by enumerating the number of episodes of infection occurring after a documented infection with the homologous serovar. The probability of reinfection with a previously experienced serovar was compared with the probability of one infection with the serovar using the binomial one-tailed test of exact probability. Serovars with a prevalence of $<10 \%$ in the gonococcal population were analyzed only according to serotype (1A or 1B). This analysis assumes that the risk of infection with a given serovar is proportional to the probability of at least one infection with the serovar and that exposure of individual women to a given strain is uniform throughout the population. Given the sexual activity of these women (3-5 different sex partners daily), these assumptions seem valid.

\section{Results}

227 women were enrolled in the study and followed over the period March 1985-June 1986. 204 experienced one or more gonococcal infections and had strains typed, 14 women had no gonococcal infections, and 9 had no strains typed. The characteristics of the study population are shown in Table I. In most patients, follow-up was discontinuous because for varying periods of time women were not resident in Nairobi and not active as prostitutes. As previously reported, a high proportion of women were positive for antibody for HIV; $65 \%$ of women enrolled in the study were HIV positive (HIV antibody status was unknown in four). The relationships between HIV status and the variables shown in Table I have been discussed elsewhere (16). The number of biweekly follow-up visits
Table I. History of Prostitution, Sexual Activity, and HIV Infection in the Study Population

\begin{tabular}{lccl}
\hline & $\begin{array}{c}\text { HIV positive } \\
(n=147)^{*}\end{array}$ & $\begin{array}{c}\text { HIV negative } \\
(n=78)^{*}\end{array}$ & \\
\hline $\begin{array}{l}\text { Age (mean years } \pm \text { SD) } \\
\text { Duration of prostitution } \\
\text { (mean months } \pm \text { SD) }\end{array}$ & $29.4( \pm 5.9)$ & $32.3( \pm 6.6)$ & $P<0.004$ \\
$\begin{array}{c}\text { Sex partners per day } \\
\quad( \pm \text { SD) }\end{array}$ & $45.3( \pm 40.9)$ & $69.1( \pm 56.0)$ & $P<0.005$ \\
$\begin{array}{c}\text { No. of clinic visits } \\
( \pm \text { SD) }\end{array}$ & $7.9( \pm 5.8)$ & $8.9( \pm 5.6)$ & $P=\mathrm{NS}$ \\
$\begin{array}{c}\text { No. of gonococcal } \\
\text { infections }( \pm \mathrm{SD})\end{array}$ & $3.9( \pm 3.1)$ & $3.8( \pm 3.0)$ & $P=\mathrm{NS}$ \\
\hline
\end{tabular}

* HIV status was not determined on two women.

ranged from 2 to 25 (mean 8.4 \pm 5.7 ) with $0-15$ episodes of gonococcal infection detected.

We tested the study hypothesis by comparing the observed data with the following four logical predictions of the study hypothesis: $(a)$ if strain-specific immunity to $N$. gonorrhoeae occurs with resultant immunity of the population, the gonococcal population infecting the study population should change rapidly with time; $(b)$ if immunity develops after gonococcal infection, women who have HIV infection and are immunodeficient should be at higher risk of infection with previously experienced strains (the mean T4/T8 ratio among a subgroup of HIV-positive women from the entire cohort was 0.93 at the outset of this study) (16); (c) the frequency of gonococcal infection should be inversely related to the duration of prostitution, because women who have been prostitutes longer have experienced a greater proportion of the current and entire gonococcal strain population; and $(d)$ women who are infected with a particular gonococcal strain will be less likely to experience a second infection with that strain than women who have not experienced that strain.

In Table II, the prevalence of gonococcal serovars isolated from the entire prostitute cohort (595 women) over the study period is shown. Four strains (1A4, 1A6, 1B3, and 1B5) accounted for $70 \%$ of all serovars over the initial $7 \mathrm{mo}$ of the study. The gonococcal population had changed dramatically by the second half of the study period. A few serovars continued to account for over two-thirds of the study population but these had changed substantially in frequency. 1B-1 and 1B-5 serovars dramatically increased in frequency, while 1A-4 and 1B-3 strains significantly decreased in frequency. Only 1A-6 strains remained at a stable prevalence over the study period. The changes in the gonococcal population were highly statistically significant. In comparing antimicrobial resistance patterns in the two halves of the study, two trends were observed. The frequency of $\beta$-lactamase-producing strains decreased from 65 to $52 \%(P<0.009)$ and the frequency of the membrane type resistance phenotype (erythromycin minimum inhibitory concentration [MIC] ${ }^{1}>2$, tetracycline $\mathrm{MIC}>2$, and Triton X MIC $>4,000 \mu \mathrm{g} / \mathrm{ml})$ increased from 23 to $41 \%$ (P

1. Abbreviations used in this paper: CI95, 95\% confidence interval; MIC, minimum inhibitory concentrations; $R R$, risk ratio. 
Table II. Changes in the Gonococcal Population Infecting the Cohort over the Study Period

\begin{tabular}{|c|c|c|}
\hline & $\begin{array}{c}\text { Initial } 7 \mathrm{mo} \\
(n=402)\end{array}$ & $\begin{array}{l}\text { Second } 7 \text { mo } \\
(n=334)\end{array}$ \\
\hline \multicolumn{3}{|l|}{$1 \mathrm{~A}$} \\
\hline 2 & $5.0^{*}$ & 2.1 \\
\hline 3 & 3.0 & 7.5 \\
\hline 4 & 21.6 & 11.1 \\
\hline 5 & 1.7 & 2.4 \\
\hline 6 & 28.1 & 24.9 \\
\hline Other & 2.0 & 1.5 \\
\hline \multicolumn{3}{|l|}{$1 \mathrm{~B}$} \\
\hline 1 & 7.2 & 18.6 \\
\hline 3 & 11.7 & 5.4 \\
\hline 5 & 10.4 & 20.1 \\
\hline 6 & 3.2 & 0.6 \\
\hline 7 & 3.5 & 0.9 \\
\hline Other & 2.5 & 2.1 \\
\hline & \multicolumn{2}{|c|}{$P<10^{-6 \ddagger}$} \\
\hline
\end{tabular}

* Figures are the percentage of the entire population isolated during each 7-mo period.

* The numbers of gonococcal isolates in each protein 1 serovar in the two time periods were compared using the chi-square test.

$<10^{-6}$ ). As no antibiotics that would be expected to select for these changes were used for treatment of gonococcal infections in this population and most antibiotic use in the population was for sexually transmitted disease treatment, the changes in the gonococcal population do not appear to be a result of antibiotic selection occurring as a result of study treatment.

Table III shows the number of infections and reinfections, homologous serovar, and homologous strain reinfections in the study group. A total of $\mathbf{4 9 7}$ gonococcal infections occurred and were available for analysis. 312 met the definition of reinfection. Because HIV status might have an important influence on the results, the analysis is stratified by HIV antibody status. Women who were HIV negative were less likely to have gonococcal infections; the mean rate of gonococcal infection was $0.56 \pm 0.03$ in HIV-positive women and $0.46 \pm 0.04$ in HIV-negative women $(P<0.05, t$ test $)$. However, we did not

Table III. HIV and Risk of Reinfection: A Comparison of HIV Antibody Status and Overall Risk of Gonococcal Infection, Risk of Same Serovar Reinfections, and Risk of Same Strain Reinfections

\begin{tabular}{lccl}
\hline & $\begin{array}{c}\text { HIV positive } \\
(n=147)\end{array}$ & $\begin{array}{c}\text { HIV negative } \\
(n=78)\end{array}$ & \\
\hline $\begin{array}{c}\text { Gonococcal infection } \\
\text { rate (number of } \\
\text { culture-positive visits } \\
\text { per number of visits) }\end{array}$ & $0.56 \pm 0.03$ & $0.46 \pm 0.04$ & $P<0.05$ \\
$\begin{array}{c}\text { Episodes of reinfection } \\
\text { Episodes of reinfection } \\
\text { with a previously } \\
\text { experienced serovar }\end{array}$ & 195 & 117 & $P=N S$ \\
$\begin{array}{c}\text { Episodes of reinfection } \\
\text { with a previously } \\
\text { experienced strain }\end{array}$ & 51 & 27 & $\begin{array}{l}P=N S \\
R R=0.26^{*}\end{array}$ \\
\hline
\end{tabular}

Table IV. The Relationship between Duration of Prostitution and the Rate of Gonococcal Infection

\begin{tabular}{|c|c|c|}
\hline Duration of prostitution* & \multicolumn{2}{|c|}{ Gonococcal infection rate } \\
\hline mo & \multicolumn{2}{|c|}{ No. infections per no. visits } \\
\hline$(n=36)$ & $0.68 \pm 0.27$ & \multirow{3}{*}{$\begin{array}{l}P<0.01^{\ddagger} \\
P<0.01\end{array}$} \\
\hline $13-48(n=84)$ & $0.51 \pm 0.24$ & \\
\hline$(n=59)$ & $0.41 \pm 0.22$ & \\
\hline
\end{tabular}

* This information was not available on 48 women.

${ }^{\ddagger} t$ test

observe a difference in the overall risk of reinfection between HIV-positive and -negative women. There was no difference in the overall frequency of reinfections with previously experienced serovars between HIV-positive and -negative women. However, when the risk of reinfection with a previously experienced strain was considered, women who were HIV negative were fourfold less likely to experience multiple infections with the same strain (risk ratio $[R R]=0.26,95 \%$ confidence interval $[$ CI95\%] $=0.07-0.88, P=0.024)$.

The relationship between the duration of prostitution and risk of gonococcal infections is shown in Table IV. As predicted by the hypothesis, more experienced prostitutes had fewer gonococcal infections. There was a stepwise decrease in the rate of gonococcal infection per visit when the duration of prostitution was stratified as 12 mo or less, $13-48 \mathrm{mo}$, and greater than $48 \mathrm{mo}$.

We performed a stepwise logistic regression analysis of the frequency of gonococcal infection entering, sex partners per day, HIV status, and duration of prostitution as dependent variables. Age was highly correlated with the duration of prostitution and was not entered in the model for this reason. HIV-positive women and women who had been prostitutes for a shorter period of time were at higher risk of gonococcal infection $(\beta=0.0945 \pm 0.0451, P<0.05$, and $\beta$ $=-0.003 \pm 0.008, P<0.00$, respectively). Sex partners per day was not related to risk of gonococcal infection.

The risk of more than one infection with a given serovar was determined as follows. The probability of infection with a given serovar was determined from the number of women who experienced one or more infections with the serovar. The exact probability of a second infection with the serovar was then determined from the number of subsequent homologous serovar reinfections using the binomial one-tailed test. The results of this analysis are shown in Fig. 1. Serovars accounting for $>10 \%$ of the gonococcal population are shown individually. Rare serovars are grouped by serotype (1A or 1B) for analysis. A strong and consistent reduction in risk of infection with the index serovar after an initial infection was shown for 1A-4, 1A-6, grouped rare $1 \mathrm{~A}$ serovars, 1B-3, 1B-5, and grouped rare $1 B$ serovars. The reduction in risk of infection varied from 2to 10 -fold $(R R=0.51-0.08)$. This was not true of serovar 1B-1.

Because the classification of some infections as treatment failures could bias the results, we analyzed the results in the manner described above with the inclusion of isolates classified as treatment failures. The exact probabilities of the observed number of reinfections were $1 \mathrm{~A}-4<0.001,1 \mathrm{~A}-6$ 


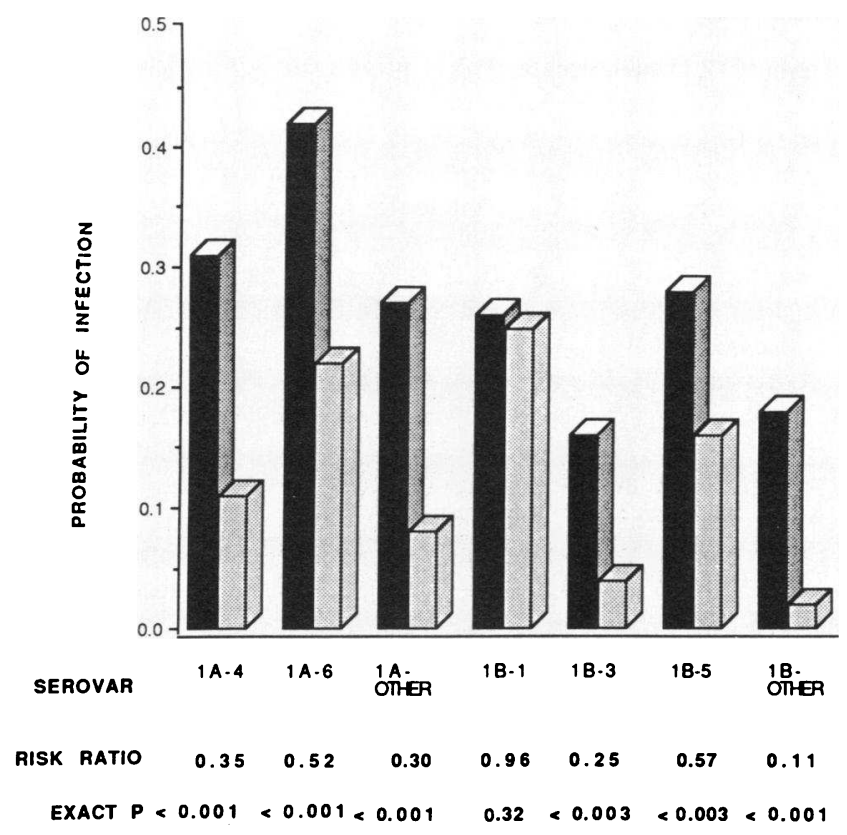

Figure 1. The risk of reinfection with an homologous serovar. Solid bars show the proportion of the entire gonococcal population for each serovar. Shaded bars show the frequency of reinfection with the homologous serovar. The probability of a homologous serovar reinfection among all reinfections occurring in women having at least one infection with a given serovar is compared with the probability of the occurrence of at least one infection by the binomial one-tailed test.

$<0.001,1 \mathrm{~A}$ other $<0.001,1 \mathrm{~B}-1=0.42,1 \mathrm{~B}-3<0.003$, and $1 \mathrm{~B}-5<0.005$.

The 1B-1 serovar is so named because it was the most frequent $1 \mathrm{~B}$ serovar in the collection on which the typing system is based (15). Thus, it may itself be a very diverse group. The 1B-1 serovars in our population are analyzed further in Table V. When classified by serovar, auxotype, and $\beta$-lactamase plasmid, 1B-1 segregates into six distinct strains. However, further analysis of reinfections with 1B-1 strains showed no reduced frequency of homologous strain reinfections.

Overall, considering the five most common serovars, the total of serovar-specific subsequent infections was 534, of which only 64 were with a previously experienced serovar. These five serovars comprised $80 \%$ of the entire gonococcal population. In addition, as shown in Table VI, the majority of same serovar reinfections occurred with nonidentical strains.

Table V. Strain Types Comprising the 1B-1 Serovar in the Study Gonococcal Population from the Study Group

\begin{tabular}{lllllll}
\hline & \multicolumn{5}{c}{ Auxotype } \\
\cline { 2 - 7 } & \multicolumn{3}{c}{ Prototrophic } & \multicolumn{4}{c}{ Proline auxotype } \\
\hline$\beta$-Lactamase plasmid* & 0 & 3.2 & 4.4 & 0 & 3.2 & 4.4 \\
Number of isolates & 7 & 4 & 4 & 10 & 2 & 17
\end{tabular}

* The numbers refer to the molecular mass of the $\beta$-lactamase plasmid in megadaltons; 0 indicates no $\beta$-lactamase plasmid.
Table VI. Analysis of Homologous Serovar Reinfection Strains

\begin{tabular}{cccc}
\hline & $\begin{array}{c}\text { Total number } \\
\text { of strains }^{*}\end{array}$ & Identical strain & Nonidentical strain \\
\hline 1A-4 & 6 & 5 & 4 \\
1 A-6 & 6 & 6 & 17 \\
1 B-1 & 5 & 6 & 10 \\
1 B-3 & 6 & 0 & 2 \\
1B-5 & 7 & 4 & 10 \\
\hline
\end{tabular}

The number of homologous serovar reinfections that occurred with identical strains (same serovar, auxotype, $\beta$-lactamase plasmid) and with nonidentical strains is shown.

* A strain is defined as a unique serovar, auxotype, $\beta$-lactamase plasmid-type class. As only two auxotypes have a significant prevalence (proline requiring and nonrequiring) and there are three $\beta$-lactamase plasmid types, the number of strains is close to six for each serovar.

\section{Discussion}

The four predictions of the study hypothesis that we tested in this study were all consistent with strain-specific immunity developing after a gonococcal infection. The gonococcal population infecting a group of female high frequency transmitters changed dramatically and rapidly with time. Women who were HIV positive and, presumably, immune deficient, experienced more frequent gonococcal infections and were at higher risk of homologous strain reinfection. The duration of prostitution showed an inverse relationship to the frequency of gonococcal infection. The most convincing evidence is that for four of five serovars considered, infection with a specific gonococcal serovar resulted in a sharply reduced risk of subsequent infection with that serovar. Among the five most frequent serovars, same serovar reinfections occurred in 64 of 534 subsequent gonococcal infections, while these serovars accounted for nearly $80 \%$ of the gonococcal population infecting the study group. Clearly, in this group of women, one infection with a given serovar substantially reduces the risk of subsequent infection with the same serovar by whatever mechanism.

One possible explanation is that the gonococcal population is undergoing change for reasons unrelated to immunity of the host, such as selective pressure by antimicrobial agents. This seems unlikely in that the changes we observed were seemingly independent of the antimicrobial agents in use for treatment of gonococcal infections in the community. Also, the effect of changes in the gonococcal population occurring for unknown reasons should be minimized by the method of data analysis. We considered the probability of reinfection with a given serovar to be the proportion of women with one or more infections with the serovar. Thus, we believe we are observing specific protection against reinfection with homologous serovars.

The nature and specificity of the putative immune response are obviously not clear from this study. It does seem probable, however, that the major antigen specificity is either protein 1 itself or closely linked to protein 1 . Protein 1 would be a likely candidate for the target of a protective immune response. It is the major protein antigen present on the surface of the gonococcus, antibody to protein 1 is elicited during infection, and antibody to protein 1 can be shown to be bacte- 
ricidal (3). Furthermore, Buchanan et al. have shown that second episodes of acute gonococcal salpingitis are uncommonly caused by the gonococci of the same protein 1 type (17). Alternatively, the protein 1 serovar may only be a marker for some other antigen to which it is closely linked, such as the gonococcal pilus. Pilus may seem somewhat unlikely as a candidate antigen for natural protective immune response given the great variability observed in pili isolated from even one strain of $N$. gonorrhoeae. However, if a strain of $N$. gonorrhoeae has a finite repertoire of pilus antigens to present to a host, and most or all of these are presented during infection, and protein 1 serovar is a marker for this antigenic repertoire, then pilus could be the antigenic target of protective immunity.

As the protective effect we observed was not absolute nor did it apply to all serovars studied, it is obviously not a completely impenetrable defense against a gonococcus of the same serovar. Several explanations for the incompleteness of this putative immune response are possible. The use of serovar as the sole basis for classifying $N$. gonorrhoeae in this study results in a relatively diverse group of organisms being classified together. We did this intentionally to more rigorously test the hypothesis, but it may have the effect of obscuring protection occurring at the level of individual strains. Some serovars (or strains) might be more immunogenic. Perhaps the exception of $1 \mathrm{~B}-1$ could be explained in this manner. The nature of the initial infection could play a role in the development of immunity; for instance, invasive infection could elicit a more vigorous and protective immune response than less severe infections. The duration of infection could, as well, be important in determining the immune response. Further studies will be necessary to explore these possibilities.

The finding that serovar-specific immunity occurs after natural gonococcal infection has important implications. The most immediate is an increased understanding of the ecologic relationship between the gonococcus and its human host. Increased understanding of the immune response to natural gonococcal infection could lead to the development of new approaches to gonococcal vaccine development. However, further work is necessary to prove that the observed protective effect is due to an immune mechanism and to characterize the specificity and duration of the protective effect.

\section{Acknowledgments}

The authors are indebted to Professor H. O. Pamba, Dr. P. Waiyaki, Professor M. Mugambi, the Ministry of Health, Republic of Kenya, and Ms. Carol Sigurdson for assistance with this work.

Dr. Plummer is the recipient of scholarships from the Medical Research Council of Canada and the Canadian Health and Life Insurance Association. Dr. Simonsen is the recipient of a Centennial Fellowship from the Medical Research Council of Canada. This work was supported by a grant from the Medical Research Council of Canada (MA-9258).

\section{References}

1. Hook, E. W., III, D. A. Olsen, and T. M. Buchanan. 1984. Analysis of the antigen specificity of the human serum immunoglobulin $\mathbf{G}$ immune response to complicated gonococcal infection. Infect. Immun. 43:706-709.
2. Lammel, C. J., R. L. Sauet, P. A. Rice, J. S. Knapp, G. K. Schoolnik, D. C. Heltbron, and G. F. Brooks. 1985. Antigen-antibody specificity in the immune response to infection with Neisseria gonorrhoeae. J. Infect. Dis. 182:990-1001.

3. Kohl, P. K., and T. M. Buchanan. 1985. Serotype specific bactericidal activity of monoclonal antibodies to protein 1 Neisseria gonorrhoeae. In The Pathogenic Neisseriae. G. K. Schoolnik, G. F. Brooks, S. Falkow, C. E. Frasch, J. S. Knapp, J. A. McCutchan, and S. A. Morse, editors. American Society for Microbiology (ASM), Washington, DC. 442-444.

4. Buchanan, T. M., and W. A. Pearce. 1976. Pili as a mediator of the attachment of gonococci to human erythrocytes. Infect. Immun. 13:1483-1489.

5. Brinton, C. C., S. W. Wood, and A. M. Brown. 1982. The development of a neisserial pilus vaccine for gonorrhoea and meningococcal meningitis. In International Symposium on Bacterial Vaccines. J. B. Robbins, J. H. Hill, and J. C. Sadoff, editors. Thieme-Stratton Inc., New York. 140-150.

6. Danielson, D., S. Bygdeman, and I. Kallings. 1983. Epidemiology of gonorrhoea: serogroup, antibiotic susceptibility and auxotype patterns of consecutive gonococcal isolates from ten different areas of Sweden. Scand. J. Infect. Dis. 15:33-42.

7. Catlin, B. W. 1973. Nutritional profiles of Neisseria gonorrhoeae, Neisseria meningitidis and Neisseria lactamica in chemically defined media and the use of growth requirements for gonococcal typing. J. Infect. Dis. 128:178-194.

8. Plummer, F. A., L. J. D'Costa, H. Nsanze, L. Slaney, W. E. DeWitt, J. S. Knapp, J. Dillon, W. L. Albritton, and A. R. Ronald. 1985. Development of endemic penicillinase producing Neisseria gonorrhoeae in Kenya. In The Pathogenic Neisseriae. G. K. Schoolnik, G. F. Brooks, S. Falkow, C. E. Frasch, J. S. Knapp, J. A. McCutchan, and S. A. Morse, editors. American Society for Microbiology (ASM), Washington, DC. 101-106.

9. Yorke, J. A., H. W. Hethcote, and A. Nold. 1978. Dynamics and control of transmission of gonorrhea. Sex. Transm. Dis. 5:31-36.

10. Rothenberg, R. B. 1983. The geography of gonorrhea: empirical demonstration of core group transmission. Am. J. Epidemiol. 117:688-694.

11. Plummer, F. A., and R. C. Brunham. 1987. Gonococcal recidivism, diversity and ecology. Rev. Infect. Dis. 9:846-850.

12. Gotschlich, E. C. 1984. Development of a gonorrhoea vaccine: Prospects, strategies and tactics. Bull. WHO. 62:671-680.

13. D'Costa, L. J., F. A. Plummer, I. Bowmer, L. Fransen, P. Piot, A. R. Ronald, and H. Nsanze. 1985. Prostitutes are a major reservoir of sexually transmitted diseases in Nairobi, Kenya. Sex. Transm. Dis. 12:64-67.

14. LaScolea, L. J., Jr., and F. E. Young. 1974. Development of a defined medium for the growth of Neisseria gonorrhoeae. Appl. Microbiol. 28:70-76.

15. Knapp, J. S., M. R. Tam, R. C. Nowinski, K. K. Holmes, and E. G. Sandstrom. 1984. Serologic classification of Neisseria gonorrhoeae with use of monoclonal antibodies to gonococcal outer membrane protein 1. J. Infect. Dis. 150:44-48.

16. Kreiss, J. K., D. Koech, F. A. Plummer, K. K. Holmes, M. Lightfoote, P. Piot, A. R. Ronald, J. O. Ndinya-Achola, L. J. D'Costa, P. Roberts, E. N. Ngugi, and T. C. Quinn. 1986. AIDS virus infection in Nairobi prostitutes: extension of the epidemic to East Africa. $N$. Engl. J. Med. 314:414-418.

17. Buchanan, T. M., D. A. Eschenbach, J. S. Knapp, and K. K. Holmes. 1980. Gonococcal salpingitis is less likely to recur with Neisseria gonorrhoeae for the same principal outer membrane protein antigenic type. Am. J. Obstet. Gynecol. 138:978-980. 